as commonly manifested, were present in this case and to a less degree in that of the other girl. Opposed to these symptoms, however, are the facts of the patient's robust health, the absence of paroxysm and of marked peculiarities of sensation and motion, the futility of restraint or coercion, and, above all, the preceding depression, with delusions, from which this condition seemed to have arisen.

Throughout the class of patients who inherit neuropathic temperaments there is a gradual evolution, as it were, of certain of the forms of mental impairment ranging from an enervation of will down to complete loss of self-control. The amount of this inherited nervous instability may be sufficient in one case to produce only a nild form of hysteria, while in another moral insanity may be the result, especially in young people. Now between the extremes come the doubtful or mixed cases, rendered additionally difficult of diagnosis by the absence of delusions. In our cases the room for doubt in this matter is small, but one often hears of similar cases of a less clear variety in which there has been considerable uncertainty whether aggravated hysteria or moral insanity is to be dealt with. 'The possibility of recovery or of improvement in the former without the stigma attached to treatment in an asylum calls for the closest inquiry into the personal and family history of these doubtful cases before yielding to the temptation of a speedy commitment. Dr. Savage says : "We have (at Bethlehem) such cases exhibiting every variety of mental symptoms. One will refuse to walk without crutches, another will insist on being fed, one will remain always like a well-dressed doll expecting to be noticed, but occupying herself with nothing but self-complacent retrospection. One will eat rubbish, another will read with the book upside down. Many such cases find their way into asylums, by a sad misfortune, I think."

Now as to prognosis. The return to and continuance in naturai bodily and mental soundness made by three of these cases after most protracted excitement points to similar occasional results in " moral insanity." Recovery is most likely to take place where the hereditary taint is slight, and where the hysterical element is prominent.

In regard to treatment the cases teach us to take into consideration the likelihood of extreme change following depression, especially in the young, and to combat the premature removal of such patients from asylum care and treatment.

Little or no benefit can come from home treatment in extreme and decided cases of moral perversion due to insanity. It is wise for the physician, before committing the patient, to fortify himself with facts gathered from several sources, to insure an accurate certificate, for in these cases of insanity more than in all others an intimate knowledge of all the facts and their intelligent statement will go far toward securing the confidence of the relatives and the comfort and proper treatment of the patient in the asylum. It may also prevent any future embarrassment to the physician from the complaints and accusations of such patients. In the asylum a course of judicious neglect has been found to be best suited to such cases.

- Coffee as a vehicle for quinia is again advocated by a writer in the Lancet. It has been shown that the taste of the drug is thus disguised only because its solubility is diminished.
PUERPERAL FEVER, PROPHYLAXIS IN HOSPITAL PRACTICE, NO'TES ON THE LYING-IN HOSPITALS OF VIENNA, DRESDEN, AND PRAGUE.

BY F. H. LOMBARD, M. D.

Among the advances made by obstetric science in the last twenty years, the recognition of the true nature of so-called "puerperal fever" and the discovery of means for preventing its appearance and dissemination are worthy of emphatic mention.

However varied and antagonistic the views held by distinguished leaders of the English, French, and German schools have been in the past, the accepted opinion of to-day is that this much-dreaded disease in all its varied forms, and with widely-differing manifestations, material is in every case due to the absorption of septic which has been introduced either from without (external infection) or from within (self-infection).

To Semmelweis is due the credit of having discovered (1847) the real cause of " puerperal fever," and his book ${ }^{1}$ embodying the result of the most careful observations while physician in the lying-in hospitals at Vienna and Pesth is to-day, after years of contention, accepted as the highest authority on this much disputed question.

The parts exposed to absorption are the internal surface of the uterus, which, as proved by Friedländer's investigations, ${ }^{2}$ is during labor deprived of its epithelium, and for at least three weeks after delivery presents a raw, wounded surface ; further, the cervical and vaginal walls, the external genitals, and the perinæum, whenever the surfaces of these are abraded.

In a vast majority of cases the infection is brought from without by the examining finger, catheter, sponge, bed-linen, etc., impregnated with organic material, from any source whatsoever, in a state of decomposition. Self-infection may take place through the absorption of portions of the placenta or membranes, of coagula, or of lochial discharges, which retained in utero have undergone decomposition; also through soft parts, which from pressure during prolonged or instrumental labor have become necrosed.

No stronger proof can be brought in support of the correctness of this view of the atiology of " puerperal fever" than the marked decrease in the mortality of lying-in women which has followed wherever it has been accepted and appropriate prophylactic measures against the dreaded disease have been strenuously carried out.

In the hope that it will not be without interest to the readers of the JounNal to review briefly the progress made in this direction in some of the lying-in hospitals of the Continent, the writer has collected the following facts from notes taken during bis residence in the hospitals at Vienna, Dresden, and Prague :-

For the ten years (1812-1822) previous to the introduction of anatomico-pathological studies in Vienna, the rate of deaths from puerperal fever in the lying-in hospital was 1.8 per cent. During the year 1823 , when medical students for the first time were obliged to dissect and to make post-mortem examinations, the mortality from puerperal fever rose with a leap to 7.5 per cent., and from that time until 1847, when Semmelweis intro-

1 Die Etiologie, der Begriff und die Prophylaxis des Kindbettfiebers. Pesth. Wien und Leipzig, 1861.

2 Carl Friedländer. Physiolog-Anatomische Untersuchungen über den Uterus. Leipzig. 
duced his rules for disinfecting hands, instrum ents, etc. with calcium hypochloricum, the death-rate never fell below 2.2 per cent. ; it averaged for these forty-five years 6.2 per cent., and in 1842 reached the formidable height of fifteen per cent., every sixth or seventh woman confined dying from puerperal fever.

The introduction of disinfectants brought the deathrate for 1848 again down to 1.3 per cent., and since that time it has never risen above two per cent., except during the interval from 1852 to 1857 , when disinfectiou was for the time abandoned (its efficacy being questioned), and, significantly enough, the death-rate rose again as high as nine per cent.

In 1865 carbolic acid was introduced, and from that time to the present antiseptic precautions have been observed with constantly increasing vigilance, and with correspondingly gratifying results. With an average of more than 10,000 births a year the mortality from puerperal fever for the last five years in the Vienua Lying-in Hospital has been under 0.75 per cent.

At Prague the results are not less striking. For the ten years (1865-1875) previous to the building of the new hospital the death-rate from puerperal fever averaged 6.67 per cent. per annum ; maximum 11.6 per cent. ; minimum 3.08 per cent.

Since the completion of the new hospital, which is a masterpiece in its hygienic arrangements, and since the introduction of antiseptic precautions rigidly observed, the number of deaths has decreased in the following remarkable ratio : -

\begin{tabular}{c|c|c|c|c}
\hline Date. & Delivered. & Died. & Died, 1 in & $\begin{array}{c}\text { Per cent. } \\
\text { Deaths. }\end{array}$ \\
\cline { 5 - 5 } 1876 & 2627 & 30 & 87.6 & \\
1877 & 2704 & 34 & 79.5 & 1.14 \\
1878 & 2776 & 45 & 61.7 & 1.26 \\
1879 & 3010 & 11 & 273.7 & 0.68 \\
1880 & 2813 & 13 & 216.4 & 0.46 \\
1881 & 2927 & 7 & 418.1 & 0.24 \\
1882 & 2963 & 7 & $\mathbf{4 2 3 . 3}$ & 0.24 \\
\hline
\end{tabular}

The general statement has been made that these strikingly favorable results have been gradually attained through the advance made in the observance of antiseptic precautions. It remains to inquire more closely into the nature of these precautions as they are to-day observed in the hospitals under consideration.

They are briefly as follows; -

The free use of soap and the nail-brush with a four per cent. solution of carbolic acid before and after every vaginal examination by physician, midwife, or student. [In Carl Braun's wards a one-tenth per cent. solution of permanganate of potassa is also used for disinfecting the hands.]

Each patient has her own urinal, bed-pan, catheter, and nozzle for vaginal and intra-uterine douche, the last two being kept constantly under a four per cent. solution of carbolic acid.

No student who has had charge of a patient with a temperature over $39^{\circ} \mathrm{C}$. is allowed to examine another patient for twenty-four hours. (In Dresden he is excluded from the lying-in room for from eight to fourteen days.)

All instruments for use during or after labor are kept under a four per cent. solution of carbolic acid.

When for any reason the hand or any instrument has been introduced into the uterus to assist labor, or whenever there is, after delivery, a foul, stinking discharge as from retained placenta or membranes, de- composed coagula or the like, the cavity of the uterus is washed out with a solution of carbolic acid (two to four per cent.) until this comes away clear. Further, in Vienna in all such cases a bougie containing pulv. iodoform five grammes (rubbed up with equal parts of powdered gum arabic and starch with sufficient glycerine to give the right consistence) is introduced into the uterus.

If the douche alone is used it is repeated every twelve or twenty-four hours, according to the severity of the case. If the double treatment is employed it is repeated once in thirty-six hours, until all untoward symptoms have disappeared. ${ }^{1}$

The same treatment is carried out in the vagina whenever its walls have been subjected to injury or to unusual pressure, as from instrumental delivery or protracted second stage.

In Prague, where extraordinary precautions are taken, a carbolic vaginal douche is given immediately before and after every delivery, and every visible rent in the vaginal mucous membrane or about the valva is at once united with silk.

The writer has seen no less than forty stitches taken to unite rents in the vaginal walls of a primipara after protracted labor.

Ruptures of the perinæum, of whatever extent, are also united with silk kept submerged in a solution of corrosive sublimate 1 to 1,000 ; the edges of the wound are sprinkled with powered iodoform, and a bit of iodoform gauze is tucked into the vulva to protect the wound from the discharges. The knees are tied together, no special diet is ordered, and the patient is not disturbed until the fourth day, when the stitches are removed. Results excellent.

In from one to two hours after delivery, unless specially contra-indicated, the patient is lifted on to a stretcher and carried to a fresh bed in one of the convalescent rooms.

In the Prague Hospital these rooms all face the south, are spacious, admirably ventilated, and contain only five beds each.

The cloths which catch the discharges are constantly renewed, and the genitals are kept clean solely by irrigation, nothing but carbolized water being allowed to approach the vulva.

If " puerperal" ulcers appear in the vaginal walls or upon the external genitals, they are treated locally as follows:-

Superficial ulcerations and wounds which have not healed by first intention are sprinkled with pulv. iodoform.

Ulcers with suppurating or diphtheritic bases are covered with a wad of salicylic cotton soaked in a one per cent. solution of calcium hypochloricum, to wbich a few drops of spts. camphor have been added; changed every two hours.

Necrosed or gangrenous parts that cannot be removed are, after irrigation with carbolic, kept covered with a wad of salicylic cotton soaked in a solution of corrosive sublimate 1 to $1,000 .^{2}$

In Dresden tr. chloride of iron is largely applied.

If a case of puerperal fever in spite of the above precautions occurs in one of the wards no attempt is made to isolate the patient. In other words, the at-

I No case of iodoform poisoning has yet occurred.

2 According to Koch's investigations this solution has a germdestroying power which is only possessed by carbolic acid of the strength of at least ten per cent. It is, therefore, the most powerful antiseptic that can be employed. 
mosphere, as a carrier of the germs of puerperal infection, is believed to play an insignificant rôle.

It is no uncommon occurrence to see a patient with the severest form of puerperal fever lying between two others in the same ward who will pass through a perfectly normal convalescence after their continement; and Winckel makes the statement, corroborated by experience, ${ }^{1}$ that the spreading of the most severe form of puerperal fever in large lying-in hospitals can be stopped within a few days solely by the careful disinfection of all instruments and the exclusion of all nurses and others who examined the affected patients before they were attacked, without necessitating the closing of the hospital, more strenuous ventilation, or the opening of new wards.

The mortality among lying-in women in private practice, calculated from extensive statistics recorded in various cities of England and the Continent, is stated to be between six and seven in every thousand.

Comparing these figures with those of the Lying-In Hospital in Prague, quoted above, we find that for the last four years in this hospital the mortality has averaged 3.25 in every 1000 .

From these and the foregoing statistics we are led to make the following deductions : -

In properly constructed lying-in hospitals, where appropriate precautions are strenuously observed, the mortality can be kept within as favorable limits as in private practice; and, further, such hospitals can be thrown open for the purposes of medical instruction without prejudice to the patients.

"The "puerperal fever" of private practice is identical with that met with in hospitals, and its occurrence and dissemination are only to be avoided by the observance of the same precautions.

Finally, whatever views one may hold upon the subject of antiseptics in general, and whether one prefers to attribute such results as the foregoing to the germ-destroying properties of carbolic and kindred substances, or merely to the increased care and cleanliness which these bring in their train, the fact remains that under no other system have such satisfactory results been obtained, and this of itself should be sufficient reason for continuing to employ the same until something better is devised.

Strassburg, April 30th.

\section{YEARLY REPORT ON DENTISTRY.}

BY WILLIAM HERBERT ROLLINS.

EDUCATION.

THE importance of a regular medical education for those who practice upon the teeth is steadily gaining ground. In the East the conservative element has as yet proved too strong for any advance in this direction, but in several of the Western medical schools oral professorships have been established.

This step marks the most important advance which has ever been made in dental education. 'To those who desire to practice upon the teeth it is of vital consequence. 'To the student of general medicine its value is great, as heretofore he has not received adequate instruction about the teeth and their diseases.

\section{ANATOMY.}

There is no particularly new material in this department. Dr. Bödecker states that a continuous net-work Pathologie und Therapie des Wochenbettes, s. 354. of protoplasm traverses not only the tubuli of the dentine, but also the side branches, which are simply anastomosing canals between the larger tubes. This protoplasmic net-work on the one hand is contimued into the enamel, and on the other into the cement. This idea has never before been so clearly demonstrated, though it was taught at the Harvard school several years ago.

Contrary to the opinion held by Darwin, of a correlation between the growth of hair and teeth, a young girl now on exhibition in London as "the missing liuk," has, though covered with hair, a normal dentition.

A curious absence of teeth has been reported in the Dental Cosmos, March, 1883. The patient, a male forty-eight years old, was one of a family of eighteen. None of the other children were edentulous, though in several the teeth were partly absent. The family history showed a maternal grandmother and an uncle, both edentulous. 'The patient has eight children, some of whom have less than the normal number, but none are entirely without teeth.

DISEASES OF THE TEETH.

Caries. - No new theory has been brought forward.

Mayr, in the New England Journal for January, 1883 , states that the acid theory of decay is untenable, because if acids were the cause of the solution of the lime salts we ought to find in the cavity lime salts in combination with the acids which are supposed to cause decay, that is, lactic, acetic, tartaric, citric, etc.

He does not find such salts present; on the contrary, in the deep layers of decay, which we may suppose are best suited for examination, he finds the lime salts but little changed in amount, and still combined, as in a normal tooth, with phosphoric and carbonic acids.

Increased attention has been given to the inflammatory theory. One objection to this is that it fails to account for the decay which always takes place in pulpless teeth.

The germ theory, as a scientific observation, may be said to date from Leber and Rottenstein, the results of whose investigations were translated by Dr. Chandler in 1873. This theory has attracted much attention. Miller, in Dental Cosmos, January, 1883, states as the results of his investigations that the first stage of caries is the extraction of the lime salts by acids produced by fermentation within the mouth; the softened tissue then becomes the abode of enormous numbers of fungi. Septothrix threads are found in the supericial layers, bacilli penetrate far into the tissue, while microccoci are found up to the junction of the sound dentine. He divides caries into three stages: Decalcification, infection of the softened dentine, and putrefaction of the organic matter of the invaded tissue.

\section{DENTAL PULP.}

Very little has been added to our knowledge of the diseases of the dental pulp since the publication of the investigations of $W$ edl. During the year the subject has been studied by Dr. Bödecker."

\section{ALVEOLAR ABSCESS.}

No new methods of treatment have been proposed. The one most in favor is to inject through the root canal. When this cannot be done to fill perfectly and

$$
2 \text { Cosmos, June, } 1882 .
$$

